\title{
Studying short-term storage of expectancies with reaction time
}

\author{
E. SCOTT GELLER \\ Virginia Polytechnic Institute and State University, Blacksburg, Virginia 24061
}

\begin{abstract}
On each of 198 choice reaction time (RT) trials, subjects verbally predicted which of two possible stimuli would occur and then waited 3,7 , or $11 \mathrm{sec}$ before receiving a ready signal and the stimulus presentation. Four groups of 20 subjects each were defined by the nature of the distractor task interpolating the time between a prediction and the ready signal. These conditions were as follows: (1) No distraction task was required, (2) the subject vocally repeated the prediction until the ready signal sounded, (3) the subject counted backward by threes until the signal occurred, and (4) the subject repeated the nonpredicted stimulus until the signal occurred. Whereas RT to incorrectly predicted stimuli did not change as a function of retention interval for any group, RT to correctly predicted stimuli increased significantly as the interval increased for the count-backward and repeat-prediction groups. For the repeatnonprediction group RT to correct predictions varied inversely with the retention interval. These results were interpreted with reference to a two-process expectancy model, short-term expectancy decay vs. interference, and negative recency effects.
\end{abstract}

When subjects are required to predict the sequentially presented stimuli in a two-choice reaction time (RT) experiment, choice RT is markedly shorter when the stimulus presentation has been predicted [i.e., a correct prediction outcome (PO)] than when the stimulus has not been predicted (i.e., an incorrect PO) (e.g., Bernstein $\&$ Reese, 1965; Williams, 1966). A common explanation for this PO effect is that subjects maintain an expectancy for the stimulus predicted and process expected events faster than unexpected events (cf. Geller, 1974; Hinrichs \& Kranz, 1970). Supporting this simple expectancy interpretation of the PO effect are the observed relationships between choice RT and factors that presumably qualify the subject's stimulus predictions. For example, choice RT to correctly predicted stimuli was an inverse function of the confidence associated with the prediction (Geller \& Whitman, 1973), the probability of a correct prediction (Geller, 1974; Whitman \& Geller, 1973), and the relative frequency of the stimulus predicted (e.g., Geller \& Pitz, 1970; Geller, Whitman, Wrenn, \& Shipley, 1971).

The present research was designed to follow up an observation that the PO effect decreased as the interval between the subject's prediction and the stimulus presentation increased (Geller \& Whitman, 1972). In the Geller and Whitman study, the prediction-to-stimulus (P-S) interval was 3,7 , or $11 \mathrm{sec}$, varying randomly among the 198 trials for each subject. Geller and Whitman referred to differential short-term storage of expectancies in order to interpret their finding that RT to correctly predicted stimuli was a direct function of the

Reprints are available from the author, Department of Psychology, Virginia Polytechnic Institute and State University, Blacksburg, Virginia 24061.
P-S interval and RT to incorrectly predicted stimuli varied inversely with the P-S interval. Thus, the decrease in the PO effect as a function of the P-S interval was presumed to reflect a decay in the memory trace of the subject's verbalized expectancy over the range of intervals used.

A prominent weakness in the Geller and Whitman (1972) study was the lack of control over the subjects' cognitive activities intervening between their predictions and the stimulus presentations. For example, after making a prediction, subjects could rehearse their prediction (thus strengthening their expectation) or think about task-irrelevant items (thus weakening their expectancy); therefore, it was actually inaccurate for Geller and Whitman to infer expectancy decay rather than expectancy interference from their findings. In other words, interfering cognitions (rather than trace decay) could have caused the decrease in the PO effect over time. The present investigation attempted to control the subjects' cognitions during the P-S interval by requiring subjects to perform a particular distractor task after vocalizing their predictions.

The classic methodology for studying short-term retention and distinguishing between decay and interference explanations of forgetting was first used by Brown (1958) and Peterson and Peterson (1959). On each trial of this paradigm, the subject receives a test item to remember for later recall (e.g., a trigram), performs a distractor task for a specified retention interval (e.g., counts backward), receives a recall cue, and then attempts to recall the test item. The dependent variable is the percent of test items recalled correctly per retention interval.

Two weaknesses of the distractor paradigm have prevented unequivocal support for a decay or interfer- 
ence theory of forgetting. First, it is difficult to find a distractor task that prevents rehearsal but does not interfere with retention. Although a complex distractor prevents rehearsal, it can also interfere with retention; and a simple distractor may not prevent surreptitious rehearsal (especially since subjects know that their recall will be tested). Second, it is possible that the dependent variable in the distractor paradigm is not sufficiently sensitive to reflect some degrees of memory loss.

In the present study, the test item was the subject's stimulus prediction and the measure of retention (or forgetting) was the difference in mean RT to correctly predicted and incorrectly predicted stimuli. Since the subjects did not have to recall their predictions in order to perform the RT task, there was probably minimal surreptitious rehearsal of the stimulus prediction. Further, choice RT could be an ideal measure of short-term memory loss (or retention), given that RT has been traditionally very sensitive to changes within and without a subject and has been used widely as a reliable index of cognitive processing.

Three different distractor tasks were varied between subjects in the present experiment, and intuitive hypotheses were tested. Specifically, during the P-S interval, some subjects counted backward by threes, other subjects repeated vocally the stimulus they had predicted, and a third group of subjects repeatedly verbalized the stimulus alternative they had not predicted (i.e., the nonpredicted stimulus). Given the common assumption that counting backward by threes prevents rehearsal (and can even interfere with retention), with this distractor the PO effect was expected to decrease significantly as a function of the P-S interval. Then, assuming that the verbal repetition (i.e., rehearsal) of a stimulus prediction would enhance one's expectancy for the predicted stimulus, it was hypothesized that for the repeatprediction distractor the PO effect would increase as a function of the P-S interval. Finally, it was hypothesized that repeating the nonpredicted stimulus would interfere maximally with expectancy for the predicted stimulus and result in the smallest PO effects. Indeed, if vocalizing a stimulus increases one's expectancy (or preparedness) for that stimulus, then the repeat-nonprediction distractor task could affect a reversed PO effect. In other words, subjects in the latter group might actually come to expect the stimulus they repeatedly verbalized, rather than the stimulus they initially predicted, and thus identify nonpredicted stimuli faster than predicted stimuli.

\section{METHOD}

\section{Subjects}

The subjects were 40 males and 40 females from the introductory psychology classes at Virginia Polytechnic Institute and State University who had not previously participated in an RT experiment. Each subject was randomly assigned to one of four experimental conditions, except for the restriction that each group include 10 males and 10 females. For their participation, the students received optional resea rch credit, applied as a bonus to determine their final grade for introductory psychology.

\section{Apparatus and Procedure}

The apparatus and general procedure were similar to those used by Geller and Whitman (1972). The order of events on each of 198 RT trials were as follows: (1) The experimenter prompted the subject's stimulus prediction by illuminating the center, horizontal line $(1.3 \mathrm{~cm})$ of the seven-segment stimulus readout; (2) the subject predicted which of the two possible stimulus events would occur, a $\sqcup$ symbol (called up) or a $\Pi$ symbol (called down); (3) with the left forefinger, the subject depressed one of two spring-return levers to indicate a stimulus prediction (these prediction levers were located on a panel to the left of the subject and were separated on a vertical axis by $7.7 \mathrm{~cm}$; the uppermost lever was used to designate a prediction of $\sqcup)$; (4) the subject's depression of the prediction lever initiated a time interval of 3,7 , or $11 \mathrm{sec}$; (5) a .25 -sec "ready" buzzer occurred and initiated a fixed interval of $1.5 \mathrm{sec} ;(6)$ the $\sqcup$ or $\Pi$ symbol $(5 \times 1.3 \mathrm{~cm})$ was presented by illuminating the appropriate segments of the seven-segment stimulus readout; (7) the subject identified the stimulus presentation as quickly as possible by pulling a left- or right-hand reaction trigger. The stimulusresponse mapping relationship was balanced within experimental conditions. A digital timer measured the interval between each stimulus presentation and identification response in milliseconds (i.e., choice RT).

The sequences of stimuli and prediction-to-buzzer intervals were the same for each subject and were derived by filtering a uniform random-number generator on an IBM 370 computer system so that each of the three time intervals preceded the presentation of each stimulus alternative on 33 trials. The experimenter told the subject that the sequence of stimuli was predetermined by a punched paper tape but did not inform the subject of the stimulus probabilities. No mention was made of the varying time intervals. Further, the task instructions encouraged the subjects to concentrate on their stimulus predictions and to be both quick and accurate when making choice reactions. Ten practice trials were given, during which taskrelated questions were answered.

\section{Interpolation Conditions}

Four experimental groups of 2.0 subjects each were differentiated on the basis of the distractor activity occurring during the 3-, 7-, or 11-sec interval that intervened between each subject's stimulus prediction and the ready buzzer. For one group, a specified interpolated distractor was not required. The intervening tasks for the other three groups were as follows.

Repeat prediction. After depressing the prediction lever, the subjects repeatedly verbalized their stimulus predictions until the ready buzzer sounded. The subjects were instructed to repeat their predictions steadily at about one per second and during the 10 practice trials, the subjects were given feedback to shape a stable response rate. Most subjects maintained a relatively steady pace of 1 to 1.5 verbalizations/sec. The mode response frequencies across subjects were 3 for the 3 -sec interval, 9 for the 7-sec interval, and 14 for the 11 -sec interval.

Count backward. After the subject pressed the prediction lever, the experimenter immediately stated a three-digit number. The subject repeated the digit and from that number proceeded to count backward by threes until the ready buzzer sounded. The task instructions indicated that on some trials the subject would only have enough time to repeat the number before the ready signal, and therefore the subject should not begin to think of subtractions until after repeating the digit. The subjects were told that the experimenter would record the number of correct subtractions and that a steady counting pace of about 1 subtraction/sec should be attempted. During the 10 practice trials, the experimenter gave feedback to shape careful and steady counting. On $96 \%$ of those trials with a $3-\mathrm{sec}$ interval, the subjects were only able to repeat the number given by the experimenter before the buzzer sounded. For the other intervals, the mode response frequencies (i.e., a repetition plus subtractions) were four for $7 \mathrm{sec}$ and eight for $11 \mathrm{sec}$.

Repeat opposite. After depressing the prediction lever, the 
subjects were required to verbalize the opposite (nonpredicted) stimulus repeatedly at a stable rate until hearing the ready signal. An approximate 1-verbalization/sec rate was shaped during practice trials and, as for the repeat-prediction group, the average rate was actually about 1 verbalization/sec with mode frequencies of 3 for $3 \mathrm{sec}, 8$ for $7 \mathrm{sec}$, and 12 for $11 \mathrm{sec}$.

\section{RESULTS}

Five subjects were replaced because of their inability to accomplish the assigned intervening task and/or to make accurate stimulus identifications. For three subjects, the task was count backward; for the other two subjects, the task was repeat opposite. Inaccurate identifications represented less than $6 \%$ of any subject's data, and no subject made more than two stimulus anticipations (i.e., an RT less than $150 \mathrm{msec}$ ). The frequency of incorrect identifications did not vary reliably as a function of the prediction-to-buzzer inter$\mathrm{val}$, the distractor condition, or the outcome of the subject's prediction (correct or incorrect). That is, the 4 (distractor condition) by 3 (interval) by 2 (PO) analysis of variance for error frequency showed no significant effects (all ps $>.10$ ). The average error percentages per group were $3.9 \%, 4.0 \%, 4.4 \%$, and $4.1 \%$ for the no-interpolation, repeat-prediction, count-backward, and repeat-opposite conditions, respectively.

The appropriate RTs of each subject were classified according to the three prediction-to-buzzer intervals and the two possible prediction outcomes (correct or incorrect). The six category means were calculated for each subject, and then group means were derived for each category by averaging across the 20 subject means of each group.

The category means for each group are depicted in Figure 1; they demonstrate differential within-subjects effects of retention interval and PO for each distractor condition. The overall analysis of variance (ANOVA) was a factorial of 4 (distractor conditions) by 3 (intervals) by 2 (POs) and showed significant main effects of group $[F(3,76)=3.85, p<.05]$ and $P O \quad[F(1,76)=$ $49.53, \mathrm{p}<.001]$. The group effect was largely due to the prominently slower reactions for the repeat-opposite distractor (i.e., Panel 4 of Figure 1). Of the interactions, two were reliable: Distractor by Interval $[\mathrm{F}(2,152)=$ $3.98, \mathrm{p}<.05]$ and Distractor by Interval by $\mathrm{PO}$ $[F(6,152)=4.21, p<.01]$. To study the nature of the interactions, an ANOVA was performed for each distractor condition (i.e., each panel of Figure 1), using the error estimates provided by the overall analysis.

For the group with no intervening distractor (no interpolation), RT was prominently faster to correct than incorrect predictions, and this PO effect was only slightly larger for the shortest interval. The ANOVA indicated a main effect of PO $[F(1,76)=18.13, p<$ $.001]$ and no effect of interval or interaction $\left(\mathrm{Fs}_{\mathrm{s}}<1\right)$.

For the repeat-prediction distractors, Figure 1 depicts a decreasing effect of $\mathrm{PO}$ as a function of the number of seconds intervening between the prediction and the ready signal. Indeed, for both correct and incorrect predictions, the relationship between $\mathrm{RT}$ and interval was linear: As the time interval increased, RT to an incorrect PO decreased and RT to a correct PO increased. For this group, the ANOVA indicated a significant main effect of PO $[F(1,76)=16.03, p<.001]$, no effect of interval $(F<1)$, and a significant Interval by PO interaction $[F(2,152)=4.92, p<.01]$. A separate analysis for correct and incorrect POs indicated that the interval effect was reliable for correct predictions $(\mathrm{p}<.05)$, but not for incorrect predictions $(\mathrm{p}>.10)$.

For the count-backward condition, RT was somewhat slower than that of the two previous groups and appeared to increase generally as a function of the number of seconds intervening between the prediction and the buzzer. The PO effect was prominently larger for the 3-sec interval than for the 7- and 11-sec intervals. The ANOVA showed significant main effects of both interval $[F(2,152)=6.18, p<.005]$ and $P O[F(1,76)=14.14$, $\mathrm{p}<.001]$. In addition, the interaction was reliable $[F(2,152)=8.47, p<.001]$. The separate analysis for correct and incorrect predictions showed a main effect of interval for correct predictions $(\mathrm{p}<.01)$, but not for incorrect predictions $(\mathrm{F}<1)$.

When the intervening distractor was repeat opposite, RT was prominently slower than it was for any other condition, and only for this group was the PO effect smallest for the shortest interval. The ANOVA demonstrated a main effect of PO $[F(1,76)=4.21]$ and a significant Interval by PO interaction $[F(2,152)=3.40$, ps $<.05]$. The analysis for simple effects showed a reliable effect of interval for correct predictions $(p<.01)$, but not for incorrect predictions $(\mathrm{F}<1)$.

\section{DISCUSSION}

Several of the findings were unexpected, but they did support the utility of the RT paradigm for studying short-term

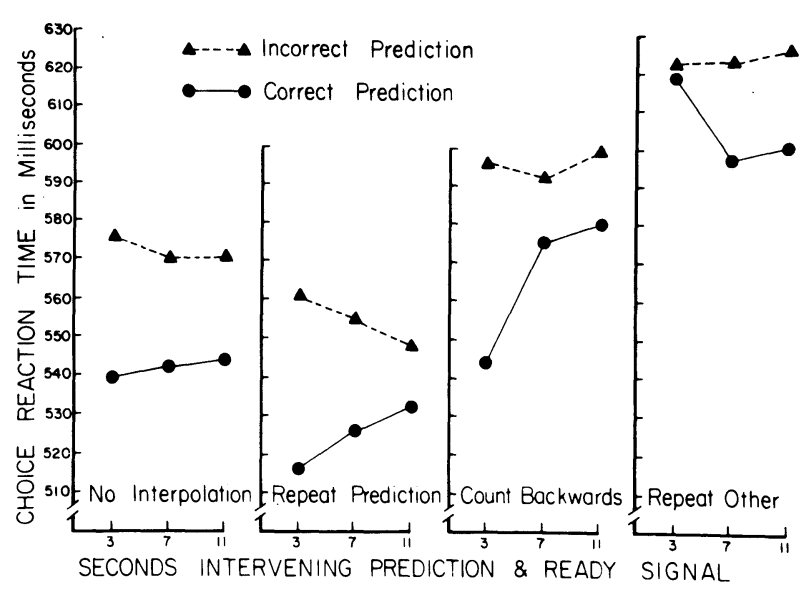

Figure 1. Choice reaction time as a function of prediction outcome (correct vs. incorrect prediction), distractor task (no interpolation, repeat prediction, count backward, or repeat other), and retention interval $(3,7$, or $11 \mathrm{sec}$ intervening between the subject's prediction and the ready signal). 
retention of expectancies. The sensitivity of choice RT as an index of forgetting was shown by the differential interactions between $\mathrm{PO}$ and the retention interval for the experimental conditions. First, the absence of a reliable decrease in the PO effect as the retention interval increased for subjects given no interpolated distractor task was surprising and contrary to the results of Geller and Whitman (1972). However, there was a procedural difference between the two studies that may account for the discrepancy. Specifically, in the earlier investigation, the subjects made a prediction and then waited 3,7 , or $11 \mathrm{sec}$ for the stimulus presentation, whereas the subjects in the nointerpolation condition of the present study gave their prediction, waited 3,7 , or $11 \mathrm{sec}$, and then received a "ready" buzzer prior to a 1.5 -sec delay and the stimulus presentation. Perhaps the ready signal in the present study served to remind the subjects of their stimulus prediction, thereby reinstating (or strengthening) a stimulus expectancy and maintaining the PO effect.

Referring to the effects of the three interpolated distractor tasks on choice RT, it is noteworthy that in each case only RT to correctly predicted (not incorrectly predicted) stimuli was significantly influenced by the retention interval (i.e., the number of seconds intervening between the prediction and the ready signal). This finding implies that a change in the facilitation to identify an expected stimulus did not implicate a concomitant parallel change in inhibition to identify the unexpected stimulus. Analogous effects on correct and incorrect POs have been shown in other RT studies, suggesting a two-process expectancy model: one process for predicted (or expected) events, and the other for nonpredicted (or unexpected) events (cf. Geller, 1974). For example, when subjects indicated their confidence in each prediction, RT to correctly predicted stimuli was an inverse function of degree of confidence, but RT to incorrectly predicted stimuli was unaffected by prediction confidence (Geller \& Whitman, 1973). When the probability of a correct prediction was manipulated between subjects, RT to correct POs varied inversely with PO probability, but RT to incorrect POs did not change as a function of PO probability (Geller, 1974; Whitman \& Geller, 1973).

For the count-backward distractor, the direct relationship between retention interval and RT to correct predictions was as hypothesized. The expectancy for the predicted stimulus faded and/or was interfered with by the interpolated task of counting backward by threes. The most prominent RT increase was from the 3- to the 7-sec interval, which may be indicative of expectancy interference as a result of the subtraction task. That is, on $96 \%$ of the trials with the $3-\mathrm{sec}$ interval, the subjects only had time to repeat the three-digit number before the ready buzzer sounded; and, therefore, the $7 \mathrm{sec}$ interval marked the start of the subtraction task. For follow-up research with the present paradigm, it would be instructive to vary the difficulty of the distractor task for a series of retention intervals (e.g., count forward by ones vs. count backward by threes) and determine if RT data can distinguish between expectancy decay and expectancy interference.

The effects of the repeat-prediction and repeat-other distractor tasks on RT to correctly predicted stimuli were in directions completely unexpected, provoking speculative interpretations and a need for further investigation. Contrary to the experimental hypothesis, vocally repeating the stimulus prediction did not enhance one's expectancy for the predicted stimulus and consequently facilitate reactions to correct predictions. Instead, the direct relationship between $\mathrm{RT}$ and the retention interval for the repeat-prediction distractor implies that verbal rehearsal of the predicted stimulus actually interfered with the expectancy for that stimulus. One speculation is that repeating the prediction resulted in some sort of satiation for that stimulus and, after several repetitions, the alternative stimulus was actually expected. The process may be analogous to the classic negative recency phenomenon observed in probability learning and RT experiments (e.g., Geller et al., 1971; Jarvik, 1951; Williams, 1966).

It was hypothesized that the repeat-other distractor would interfere most with stimulus expectancy, but in fact only this task resulted in a reliable decrease in $\mathrm{RT}$ to correct predictions when the retention interval increased from 3 to $7 \mathrm{sec}$. Actually, the prominently slowest overall RT for the repeat-other group does imply marked interference as a result of the particular distractor. However, the within-subjects effects of retention interval for this condition suggest that RT to identify a correctly predicted event was more inhibited (or less facilitated) after the subject repeated the opposite (nonpredicted) stimulus for $3 \mathrm{sec}$ (i.e., about three repetitions) than when the subject repeated the nonpredicted stimulus for $7 \mathrm{sec}$ (i.e., about eight repetitions). Indeed, repeating the nonpredicted stimulus for $3 \mathrm{sec}$ eliminated the PO effect, but the PO effect reappeared when the nonpredicted stimulus was repeated for 7 or $11 \mathrm{sec}$. My explanation for this observation is highly speculative, but not unlike the interpretation of the results for the repeat-prediction condition. Thus, a few verbalizations of the nonpredicted stimulus (i.e., for $3 \mathrm{sec}$ ) interfered with the subjects' stimulus expectancy as hypothesized and eliminated the PO effect, but extended vocalizations of the nonpredicted stimulus (i.e., for 7 or $11 \mathrm{sec}$ ) resulted in "satiation" for this stimulus and an expectation for a stimulus alternation (i.e., for the nonverbalized but previously predicted stimulus).

\section{REFERENCES}

Bernstein, I. H., \& ReEse, C. Behavioral hypotheses and choice reaction time. Psychonomic Science, 1965, 3, 259-260.

Brown, J. A. Some tests of the decay theory of immediate memory. Quarterly Journal of Experimental Psychology, 1958, 10, 12-21.

GELLER, E. S. Preceding prediction outcome and prediction outcome probability: Interacting determinants of choice reaction time. Journal of Experimental Psychology, 1974, 103, 426-430.

Geller, E. S., \& Pitz, G. F. Effects of prediction, probability, and run length on choice reaction speed. Journal of Experimental Psychology, 1970, 84, 361-367.

Geller, E. S., \& Whitman, C. P. Prediction outcome and choice reaction time: A memory-dependent relationship. Journal of Experimental Psychology, 1972, 96, 334-337.

Geller, E. S., \& Whitman, C. P. Confidence in stimulus predictions and choice reaction time. Memory \& Cognition, 1973, 3, 361-368.

Geller, E. S., Whitman, C. P., Wrenn, R. F., \& Shipley, W. G. Expectancy and discrete reaction time in a probability reversal design. Journal of Experimental Psychology, 1971, 90, 113-119.

Hinrichs, J. V., \& Krainz, P. L. Expectancy in choice reaction time: Anticipation of stimulus or response? Journal of Experimental Psychology, 1970, 85, 330-334.

JARVIK, M. E. Probability learning and a negative recency effect in the serial anticipation of alternative symbols. Journal of Experimental Psychology, 1951, 41, 291-297.

Peterson, L. R., \& Peterson, M. S. Short-term retention of individual verbal items. Journal of Experimental Psychology, 1959, 58, 193-198.

Whitman, C. P., \& Geller, E. S. Prediction outcome distributions as determinants of choice reaction time. Perception \& Psychophysics, 1973, 13, 105-107.

Williams, J. A. Sequential effects in disjunctive reaction time: Implications for decision models. Journal of Experimental Psychology, 1966, 71, 665-672.

(Received for publication September 21, 1981.) 